\title{
Effects of the Global Multicultural Nursing Competency Enhancement Program on the Cultural Competence of Nursing College Students
}

\author{
Mi-Jung Choi ${ }^{1}$ and Seok-Young $\mathrm{Han}^{2 *}$ \\ Assistant Professor, Dept. Nursing Science, Chosun Nursing College, Pilmundaero, \\ Dong-gu, Gwangju, Korea \\ 'tinercmj@cnc.ac.kr, ${ }^{2 *}$ hansy4ever@cnc.ac.kr
}

\begin{abstract}
The purpose of this study was to develop the global multicultural nursing competency enhancement program (GMNCE program) and to examine its effects on the cultural competence of nursing college students. A quasi-experimental study using a nonequivalent control group pretest-posttest design was applied to an experimental group $(n=33)$ and a control group $(n=36)$. The experimental group participated in a global camp held in Malaysia for the GMNCE program for 3 weeks while the control group did not. The data, collected using self-report structured questionnaires before the program and after the intervention, were analyzed with descriptive statistics, independent t-test, $\chi^{2}$-test, and ANCOVA using SPSS 24.0 program. Compared to the control group, the experimental group reported significant positive changes in cultural competence $(F=9.71, p=.003)$ including cultural knowledge $(F=9.52, p=.003)$, cultural skills $(F=13.42, p=.001)$, cultural encounters $(F=4.67, p=.035)$, cultural awareness $(F=11.01, p=.002)$, and cultural sensitivities $(F=9.45$, $p=.003)$, and the effect of the program was also supported by significant differences between the two groups. The results of this study indicate that the GMNCE program is effective in raising the level of cultural competence of nursing students. Based on the findings, this study suggests a need to include content from cultural competence curricula or training programs to improve undergraduate nursing college students' cultural competence.
\end{abstract}

Keywords: Multicultural nursing, Cultural knowledge, Cultural skills, Cultural encounters, Cultural awareness, Cultural sensitivities, Nursing college students

\section{Introduction}

Recently, the number of foreign workers, marriage immigrants, North Korean refugees, foreign students, and midway immigrants has increased in South Korea [1]. The number of foreigners living in South Korea exceeds 1.58 million, accounting for 3.2 percent of the total population [2], and the number of foreign patients tallied at around 120,000 thanks to the government's medical tourism project as well as the revision of the medical law, which has increased the chance of meeting patients with various cultural backgrounds in medical fields and various communities [3]. Due to the impact of these social changes, reinforcing the

Article History:

Received (November 30, 2019), Review Result (January 4, 2020), Accepted (February 9, 2020) 
multicultural capacity of healthcare providers, including nurses, has become a new concern [4].

Healthcare providers must understand the cultural context, including the value and belief in the health of multicultural groups, and support them to ensure proper health care [5]. However, due to health care providers, when providing medical services to multicultural people, lack knowledge and experience on lifestyles and health behaviors according to their respective cultures [6] as well as the existence of cultural differences in health and health care [7], the satisfaction when being provided with medical services is affected, causing health inequality [8]. Thus, cultural competency, which means reducing and managing the unfair discrimination arising between contrasting cultures efficiently by improving the prejudices, discrimination, and obstacles experienced by multicultural subjects with diverse cultural backgrounds and values when being provided with health care services, is required [9].

It is necessary to confirm the various components that consist of cultural competency since it includes complicated concepts. Cultural competency signifies the acquisition of knowledge, awareness, and skills related to diverse cultural groups as well as consistent behavior, attitude, and policies of healthcare service providers to convey healthcare services effectively in multicultural situations [10]. Furthermore, cultural competency is applying nursing skills, such as assessing the health needs of the client or clinical diagnosis, to subjects with cultural sensitivities- which is to view the clients based on trust, acceptance, and respect; cultural knowledge, acquired through various ways, as well as cultural awareness, which is to verify the standards, beliefs, and values of multicultural people [11]. Caffrey, Neander, Markle, and Stewart [12] classify cultural awareness, cultural knowledge, and cultural skills as a component of cultural competence, while Schim, Benkert, Doorenbos, and Miller [13] emphasize aspects of cultural behavior, which means to provide culturally congruent nursing care through cultural awareness based on cultural sensitivities, they strive to learn cultural differences and cultural experiences to develop cultural competency. Therefore, a conclusion can be drawn that cultural knowledge, cultural awareness, cultural encounters, cultural sensitivities, and cultural skills, common elements of cultural competence, are included as the main content of the multicultural nursing curriculum to comprehensively change the knowledge, skills, and attitudes of multicultural nursing care [9][14].

Cultural competency is suggested as a core capability for nurses to provide efficient cultural nursing, and nursing college students, in particular, are required to receive systematic multicultural-related education with continuous efforts during the curriculum of the nursing education institution to acquire cultural competency as pre-specialized nursing personnel who will prevent and improve and come into contact with the health of various multicultural people in local communities, including clinical hospitals and schools [4][9][12]. It can be said that the integrated results of cultural awareness and cultural sensitivities are shown as actions in clinical practice by possessing knowledge through nursing education to correctly recognize the language, religion, and beliefs by showing an open attitude toward various cultural groups [13]. Thus, it is a task of foremost significance in a multicultural society to develop a systematic transcultural nursing education and a cultural capacity-building program aiming for the enhancement of nurses' cultural competence [15].

In Western countries, which have already experienced multicultural society, research results and education strategies are being reported by developing and operating multi-cultural nursing competency education programs within various curricula [16][17], and nursing education standards, based on numerous studies and curricula for the reinforcement of cultural competency of nurses and nursing college students, are presented for culturally 
appropriate nursing practice [18]. The American Association of Colleges of Nursing [19] establishes five core components of cultural competency for the operation of cultural competency curricula, organizing and presenting educational content such as nursing theory and nursing model for cultural competence, intramural theoretical lectures for the enhancement of cultural competency, integrated learning strategies required in clinical practice and reference material to learn various cases required within the curriculum for the acquisition of aforementioned skills.

In South Korea, Peek and Park [20] developed and operated a multicultural education program called 'Cultural Class for Nursing College Students' and found out that cultural competency, consisting of domains of cultural awareness, cultural knowledge, cultural skills, cultural desires, and cultural encounters, and self-efficacy have increased. However multicultural nursing education program that operated once for a short period is rather impractical for the enhancement of cultural competency, which leads to the conclusion that multicultural nursing education must be conducted before clinical and local community nursing practice, to approach health care and nursing issues of a multicultural society by understanding and embracing cultural diversity and cultural values. Cho Chung, Han, and Seo [21] conducted this study to investigate the effects on cultural competence by developing and applying the 'Cultural Competence Educational Program for Nursing Students' for junior nursing college students. The results showed that the multicultural nursing education program is effective in promoting cultural competence, including cultural knowledge, cultural awareness, cultural acceptance, and cultural competence behaviors of nursing college students. The study was particularly noteworthy since it provided a long-term program with content that fully covered the subdivision of cultural competence for efficient multicultural nursing education, and also since various teaching-learning strategies were applied.

Therefore, this study was conducted to operate the 'Global Multicultural Nursing Competence Enhancement program (GMNCE program)' for senior nursing college students who are prospective nurses before nursing practice in clinical fields and to evaluate the effectiveness through multicultural experiences overseas and training of global medical institutions. The purpose of this study is to verify the effect on cultural competence after operating the GMNCE program during winter vacation. Cultural competence includes five sub-domain areas of cultural knowledge, cultural skills, cultural encounters, cultural awareness, and cultural sensitivities, and the hypotheses of this study are as follows.

Hypothesis 1. The experimental group who engaged in the GMNCE program will score higher in cultural competencies than the control group not attending the program.

Supplementary hypothesis 1.1. The experimental group who engaged in the GMNCE program will score higher on cultural knowledge than the control group not attending the program.

Supplementary hypothesis 1.2. The experimental group who engaged in the GMNCE program will score higher in cultural skills than the control group not attending the program.

Supplementary hypothesis 1.3. The experimental group who engaged in the GMNCE program will score higher cultural encounters than the control group not attending the program.

Supplementary hypothesis 1.4. The experimental group who engaged in the GMNCE program will score higher on cultural awareness than the control group not attending the program.

Supplementary hypothesis 1.5. The experimental group who engaged in the GMNCE program will score higher cultural sensitivities than the control group not attending the program. 


\section{Method}

\subsection{Research design}

This study was a quasi-experimental study of the non-equivalence control group design to identify the effects on cultural competence by running the GMNCE Program for nursing college students.

\subsection{Participants}

This study was conducted in a 3rd grade C nursing college in G metropolitan. Nursing college students who participated in the GMNCE Program held in Malaysia was classified as the experimental group; those who did not attend were classified as the control group. The criteria for selecting the participants were: first, willingness to participate in the recruitment of participants in the Malaysia global camp, and second, provision of documentary consent to participate in the program with a proper understanding of the purpose and procedure of the research.

The number of study participants was calculated using the G*Power 3.1.9.2 program. Based on a significant level $(\alpha)$, a power(1- $\beta$ ) of 0.80 , and an effect size(d) of $0.30,64$ samples were calculated for independent sample verification, with 33 experimental groups and 36 control groups recruited to finally include 69 people in the data analysis.

\subsection{Measurements}

The cultural competence of nursing college students was measured using the 'Cultural Competence Scale for Nursing Students (CCS-NS)' tool developed by Han \& Cho Chung [22]. The CCS-NS is a 5-point scale tool consisting of nine questions about cultural knowledge, six questions about cultural skills, four questions about cultural encounters, four questions about cultural awareness, and four questions about cultural sensitivities, ergo 27 questions altogether. The total score of a nursing college student's cultural competence measuring tool ranges from 27 to 135, and in this study, the average score was used, thus the higher the score, the higher the degree of cultural competence. The CCS-NS reliability verification was Cronbach's alpha .91, and Cronbach's alpha .90 in this study.

\subsection{Data collection}

The data were collected from Dec. 27, 2018, to Jan. 16, 2019. The preliminary test of cultural competency for the experimental group was conducted by the researchers at the GMNCE global camp orientation, and the test for the control group was conducted by the coresearcher in the classroom of $\mathrm{C}$ College after the final examination. The self-reporting questionnaire was distributed and collected. After operating a three-week GMNCE program, we conducted cultural competency follow-up with the experimental and control groups at the same time.

\subsection{Data analysis}

The collected data were analyzed using the SPSS Statistics 24.0 program. The general characteristics of the participants of the two groups were analyzed with numbers and percentages, and the homogeneity test between the two groups for general characteristics and dependent variables was analyzed with $\chi^{2}$-test and ANCOVA. And for calculation of the 
difference between the two groups before and after the operation of the GMNCE program was analyzed through the Independent t-test.

\subsection{Ethical considerations}

For the protection of the participant's rights, the purpose of this study and its progress was explained. They were also explained that there were no side effects or hazards arising from the participation. The data collected were used only for research purposes and personal confidentiality was guaranteed. In addition, the research participation was conducted after written voluntary consent was obtained and the participants fully explained the benefits and losses of the research participation, privacy and confidentiality, and the possibility of withdrawal from the research participation at their will at any time during the study participation, and that no personal disadvantages would arise therefrom.

\section{Results}

\subsection{General Characteristics and homogeneity between the two groups}

The results of the homogeneity test for the general characteristics and pre-dependent variables of the experimental and control groups under this study showed that the general characteristics of the two groups did not differ statistically significantly and therefore that the two groups were homogeneous [Table. 1]. When it comes to cultural competence, the pretest showed significant differences between the two groups in cultural knowledge, skills, and awareness, resulting in inhomogeneity [Table. 2].

Table 1. Homogeneity of general characteristics $(\mathrm{N}=69)$

\begin{tabular}{|c|c|c|c|c|c|c|}
\hline Characteristics & Categories & $\begin{array}{c}\text { Exp. }(n=33) \\
N(\%)\end{array}$ & $\begin{array}{c}\text { Cont. }(\mathrm{n}=36) \\
\mathrm{N}(\%)\end{array}$ & $\begin{array}{c}\text { Total. }(\mathrm{n}=69) \\
\mathrm{N}(\%)\end{array}$ & $\chi^{2}$ & $\mathrm{p}$ \\
\hline \multirow{2}{*}{ Gender } & Female & $30(43.5)$ & $34(49.3)$ & $64(92.8)$ & \multirow{2}{*}{0.32} & \multirow{2}{*}{.458} \\
\hline & Male & $3(4.3)$ & $2(2.9)$ & $5(7.2)$ & & \\
\hline \multirow{3}{*}{ Age (yr) } & $\leq 20$ & $7(10.1)$ & $12(17.4)$ & $19(27.5)$ & \multirow{3}{*}{14.82} & \multirow{3}{*}{.096} \\
\hline & 21 & $22(31.9)$ & $12(17.4)$ & $34(49.3)$ & & \\
\hline & 22 & $4(5.8)$ & $12(17.4)$ & $16(23.2)$ & & \\
\hline \multirow{2}{*}{ Religion } & Have & $7(10.1)$ & $11(16.0)$ & $18(26.1)$ & \multirow{2}{*}{4.98} & \multirow{2}{*}{.174} \\
\hline & Have not & $26(37.7)$ & $25(36.2)$ & $51(73.9)$ & & \\
\hline \multirow{2}{*}{$\begin{array}{l}\text { Interaction with } \\
\text { foreigner }\end{array}$} & Yes & $14(20.3)$ & $18(26.1)$ & $32(46.4)$ & \multirow{2}{*}{0.40} & \multirow{2}{*}{.631} \\
\hline & No & $19(27.5)$ & $18(26.1)$ & $37(53.6)$ & & \\
\hline
\end{tabular}

Exp. $=$ Experimental; Cont.$=$ Control group

Table 2. Homogeneity of cultural competence ( $\mathrm{N}=69)$

\begin{tabular}{|c|c|c|c|c|}
\hline \multirow{2}{*}{ Variables } & Exp. $(\mathrm{n}=33)$ & Cont. $(\mathrm{n}=36)$ & \multirow{2}{*}{$\mathrm{t}$} & $\mathrm{p}$ \\
\cline { 2 - 5 } & $\mathrm{M} \pm \mathrm{SD}$ & $\mathrm{M} \pm \mathrm{SD}$ & & \\
\hline Cultural competence & $2.94 \pm 0.41$ & $3.09 \pm 0.45$ & 2.08 & .155 \\
\hline Cultural knowledge & $2.38 \pm 0.56$ & $2.77 \pm 0.61$ & 6.78 & $.012 *$ \\
\hline Cultural skills & $2.29 \pm 0.62$ & $2.68 \pm 0.61$ & 6.01 & $.017 *$ \\
\hline Cultural encounters & $2.70 \pm 0.80$ & $2.92 \pm 0.70$ & 1.28 & .263 \\
\hline Cultural awareness & $4.16 \pm 0.56$ & $3.81 \pm 0.78$ & 4.23 & $.044 *$ \\
\hline
\end{tabular}


Effects of the Global Multicultural Nursing Competency Enhancement Program on the Cultural Competence of Nursing College Students

\begin{tabular}{|c|c|c|c|c|}
\hline Cultural sensitivities & $4.16 \pm 0.50$ & $3.90 \pm 0.65$ & 3.10 & .084 \\
\hline
\end{tabular}

\subsection{Effect of multicultural nursing education program}

Because the two groups homogeneity in cultural competency was not ensured in the pretest results, ANCOVA analysis was conducted to test the difference between the experimental group and the control group. As a result, hypothesis 1 was supported since there was a statistically significant difference $(\mathrm{F}=9.71, \mathrm{p}=.003)$ between groups in cultural competency. And sub-hypothesis 1.1. Cultural knowledge ( $\mathrm{F}=9.52$, $\mathrm{p}=.003), 1.2$. Cultural skills $(\mathrm{F}=13.42$, $\mathrm{p}=.001)$, 1.3. Cultural encounters $(\mathrm{F}=4.67, \mathrm{p}=.035)$, 1.4. Cultural awareness $(\mathrm{F}=11.01$, $\mathrm{p}=.002)$, 1.5. Cultural sensitivities $(\mathrm{F}=9.45, \mathrm{p}=.003)$, were also supported by significant differences between the two groups [Table. 3 ].

Table 3. Effects of the GMNCE program between two groups $(\mathrm{N}=69)$

\begin{tabular}{|c|c|c|c|c|c|}
\hline Variables & Sum of Squares & df & Mean Square & $\mathrm{F}$ & $\mathrm{p}$ \\
\hline Covariate: Pre-Cultural competence & 2.175 & 1 & 2.175 & 9.71 & .003 \\
\hline Between Groups & 9.703 & 1 & 9.703 & 43.34 & .000 \\
\hline Error & 12.986 & 66 & 0.224 & - & - \\
\hline Total & 24.86 & 68 & - & - & - \\
\hline Covariate: Pre-Culture knowledge & 347.434 & 1 & 347.434 & 9.52 & .003 \\
\hline Between Groups & 703.463 & 1 & 703.463 & 19.28 & .000 \\
\hline Error & 2116.772 & 66 & 36.496 & - & - \\
\hline Total & $3,167.67$ & 68 & - & - & - \\
\hline Covariate: Pre-Cultural skills & 135.719 & 1 & 135.719 & 13.42 & .001 \\
\hline Between Groups & 488.416 & 1 & 488.416 & 48.30 & .000 \\
\hline Error & 586.543 & 66 & 10.113 & - & - \\
\hline Total & $1,210.68$ & 68 & - & - & - \\
\hline Covariate: Pre-Cultural encounters & 39.627 & 1 & 39.627 & 4.67 & .035 \\
\hline Between Groups & 319.313 & 1 & 319.313 & 37.66 & .000 \\
\hline Error & 491.790 & 66 & 8.479 & - & - \\
\hline Total & 850.73 & 68 & - & - & - \\
\hline Covariate: Pre-Cultural awareness & 72.082 & 1 & 72.082 & 11.01 & .002 \\
\hline Between Groups & 40.679 & 1 & 40.679 & 6.21 & .016 \\
\hline Error & 379.711 & 66 & 6.547 & - & - \\
\hline Total & 492.47 & 68 & - & - & - \\
\hline Covariate: Pre-Cultural sensitivities & 52.303 & 1 & 52.303 & 9.45 & .003 \\
\hline Between Groups & 81.799 & 1 & 81.799 & 14.76 & .000 \\
\hline Error & 320.882 & 66 & 5.532 & - & - \\
\hline Total & 454.98 & 68 & - & - & \\
\hline
\end{tabular}

The results showed that the GMNCE program is effective in enhancing the cultural competences of nursing college students, which includes cultural knowledge, cultural skills, cultural encounters, cultural awareness, and cultural sensitivities. The hypothesis of this study, "The experimental group who engaged in the GMNCE Program will score higher cultural competencies than the control group not attending the program," was supported with 
statistic significance. Before the operation of the GMNCE program, the pre-test score for cultural competency was 2.94, slightly lower than the findings of Han [23], which evaluated the cultural competency of nursing college students using the same tools as this study, yet higher than the score of 2.57 for the $1^{\text {st }}$ degree of nursing college students[21]. Some studies have shown that the low cultural competency scores of nurses at general hospitals and firstyear nursing students have ironically demonstrated the need to develop a curriculum for multicultural nursing courses since it demonstrated that the reason for the short history of migration and multiculturalism in South Korea, lack of experience in multicultural nursing education, and lack of contact and exposure to multicultural groups [24][25]. In addition, when comparing the average scores by sub-domain of cultural competence, cultural awareness, and cultural sensitivities scores were higher compared to cultural encounters, cultural skills and cultural knowledge, and cultural skills and cultural knowledge, whose scores were significantly lower among the total domains. This was consistent with the findings of Park [26] and Cha [27], who reported cognitive and affective openness to other cultures at a time of transition to a multicultural society, but the lack of ability to perform practical behaviors. This was different from the results of the preliminary examination of a study for the application of multicultural nursing and cultural competency program for multicultural maternity nursing care [28]. After operating the GMNCE program, the cultural competency score increased from 2.94 to 4.04 compared to the control group's score. This was consistent with the findings of a study that operated regular and non-regular curriculum and cultural experience programs for nursing college students to enhance their cultural competency [12][20]. In addition, it was also consistent with the results of a study that applied cultural competency enhancement programs in special transfer nursing student curriculum alongside a study that applied cultural competency improvement programs for multicultural maternity nursing care [14][28].

First, looking at the results of the supplementary hypothesis of cultural competency, the score of cultural knowledge of the experimental group after the application of the GMNCE program showed a significant positive increase compared to the control group. This was in line with the preceding studies in which multicultural nursing education programs enhanced the cultural knowledge of nursing college students as well as nurses [12][14][20][21][28].

Second, after applying to the GMNCE program, the degree of cultural skills of the experimental group increased statistically significantly. This was consistent with the findings of Kim [29] that demonstrated that multicultural nursing education is effective in changing behaviors related to cultural nursing. However, in the case of education through a short-term program of case-based small group learning method for multicultural maternity nursing care, the effect of cultural activities did not reach a statistically significant level due to the limitations of short-term education lasting only for six quarters [28]. Education related to cultural skills through the GMNCE program is assumed to have lacked practical nursing services as there were somewhat fewer opportunities for direct contact with subjects from other cultures, unlike cultural knowledge and cultural awareness. However, the multicultural experience of an individual, either direct or indirect, played a role not only in improving cultural competency but also in enhancing cultural sensitivities and cultural awareness through multicultural nursing education programs as well as cultural skills as a consequence of wider cultural knowledge. In other words, since it seems that a lot of in-contact experiences with diverse multicultural students and their families as well as training and education on cultural diversity would have positive effects on cultural skills by promoting cultural awareness and cultural knowledge [30], cultural experience overseas, global training programs, multi-cultural experiences in clinical practice institutions, and voluntary activities 
in-and abroad, through interaction with people from different cultural backgrounds, are expected to be effective in enhancing the cultural competency of nursing college students [4]. In this study, various teaching and learning strategies for cultural skills, such as case studies, the situation plays, interviews with multicultural subjects through field works, and group discussions were used. Various teaching strategies such as role play, discussion in small groups, demonstration, case-based learning, problem-based learning, flip-learning, action learning, and simulation-based learning should be developed for efficient multicultural nursing education in the future.

Third, after the operation of the GMNCE program, a statistically significant increase in score for cultural encounters of the experimental group compared to the control group demonstrated the effectiveness of the program for wider experiences. Cultural encounters mean the environmental factors that improve cultural competencies such as encountering foreigners, through activities like voluntary work overseas, international cultural exchange activities and travel, or engagement in multicultural-themed education. This study showed consistency with the study of Caffrey, Neander, Markle, and Stewart [12] in which advancement in cultural competency was reported after completing a multicultural education program that organized nursing college students to perform nursing action to patients from diverse cultural backgrounds as well as the study of Cooper-Brathwaite [31] which reported a reinforcement in cultural encounters after a multicultural education program operated for public health nurses.

Fourth, after the application of the GMNCE program, the statistically significant increase in score for cultural awareness of the experimental group compared to the control group confirmed the effectiveness of the program in promoting cultural awareness. It was consistent with the results of studies that employed various programs for nursing college students and nurses [25][26].

Finally, the experimental group engaged in the GMNCE program had a higher score for cultural sensitivities than the control group, which had statistically significant. In other words, it means that the GMNCE program for nursing college students was effective in enhancing receptivity to culture through cultural sensitivities. This is in line with the results of studies such as a study that proved the effectiveness of an experience program for nursing college students in increasing cultural sensitivities [14], a study which verified that the multicultural attitude including cultural sensitivity was improved through a multicultural social welfare special lecture program for college students majoring in social welfare [32] as well as a study which demonstrated an increased cultural sensitivity after a cultural competency enhancement program for the nursing of multicultural maternity [27]. Cultural sensitivities must be preceded by a change in multicultural attitude, which can be achieved with a sufficient understanding and receptive attitude between major and immigrant cultures [23]. Therefore, it is possible to make a judgment that a positive and receptive change in attitude toward cultural diversity was made possible through a multicultural nursing education program.

This study has difficulty in generalizing results since the study was conducted on nursing college students of a single university, and it also has limitations of investigation research through short-answer questions, that is, the lack of in-depth exploration. In addition, further complementary research on the sustainability of the effects of the GMNCE program through winter vacation will be needed. In addition, the point at which it will be effective to take place before and after the GMNCE program has entered the clinical practice must be proven through continuous research, and it is meaningful to apply the multicultural nursing curriculum and to implement the cultural competency of nursing college students graduating 
with sufficient personal experience. Despite these limitations, it can be seen as very urgent and important to train nurses who combine cultural capabilities in a rapidly changing internationalizing society into a multicultural society. Therefore, various educational programs to enhance the cultural competency of nursing college students in the future are developed and operated to provide culturally suitable nursing services to multicultural students.

\section{Conclusions}

This study was conducted to determine the effects of the GMNCE program on the cultural competency of nursing college students. The results of this study demonstrated that the GMNCE program was effective in the improvement of nursing college students' cultural competency. These results will let nursing college students establish and expand the role of specialized nurses capable of efficiently managing multicultural subjects by providing effective nursing intervention to multicultural subjects.

Through this study, the acquisition of cultural competencies to provide diverse and practical nursing to local multicultural subjects by operating multicultural sharing programs or culture exchange programs overseas associated with clinical practices after school and during vacation is expected to accelerate.

Repetitive research on cultural competency education programs for nursing college students is demanded, as is cross-sectional research to verify the effects of providing direct multicultural nursing services during clinical practice after the education.

\section{References}

[1] H. Y. Kang and S. Y. Han, "A study on a social distance of nursing students toward minority groups in a metropolitan city,” Journal of Korean Public Health Nursing, vol.27, no.1, pp.166-178, (2013)

[2] Korea Immigration Service, "Korea immigration service statistics 2014," Gwacheon: Ministry of Justice, (2014)

[3] Ministry of Public Administration and Security, "Statistics on foreign residents by local governments in 2012," (2012)

[4] W. O. Oh, "Factors influencing cultural sensitivity among nursing students," Journal of Korean Academy of Child Health Nursing, vol.17, no.4, pp.222-229, (2011)

[5] J. Campinha-Bacote, "The process of cultural competence in the delivery of healthcare services: a model of care,” Journal of Transcultural Nursing, vol.13, no.3, pp.181-184, (2002)

[6] J. W. Kim, "Health status and medical service utilization of migrant workers in Korea," M.S. thesis, Yonsei University, (2008)

[7] C. K. Koh and S. K. Koh, "Married female migrants' experiences of health care services," The Journal of Korean Academic Society of Nursing Education, vol.15, no.1, pp.89-99, (2009)

[8] S. J. Cha, "Unmet health care needs of marriage migrant women in Korea," M.S. thesis, Seoul National University, (2012)

[9] J. Campinha-Bacote, "Becoming culturally competent in ethnic psychopharmacology," Journal of Psychosocial Nursing and Mental Health Services, vol.45, no.9, pp.27-33, (2007)

[10] T. L. Cross, B. J. Bazron, K. W. Dennis, and M. R. Isaacs, "Towards a culturally competent system of care: a monograph on effective services for minority children who are severely emotionally disturbed," Washington, DC: CASSP Technical Assistance Center, Georgetown University Child Development Center

[11] I. Papadopoulos, "The papadopoulos, tilki and taylor model of developing cultural competence in nursing," Journal of Health, Social, and Environmental Issues, vol.4, no.1, pp.5-7, (2003) 
[12] R. A. Caffrey, W. Neander, D. Markle, and B. Stewart, "Improving the cultural competence of nursing students: results of integrating cultural content in the curriculum and an international immersion experience," The Journal of Nursing Education, vol.44, no.5, pp.234-240, (2005)

[13] S. M. Schim, A. Z. Doorenbos, J. Miller, and R. Benkert, "Development of a cultural competence assessment instrument," Journal of Nursing Measurement, vol.11, no.1, pp.29-40, (2003)

[14] M. H. Park and E. A. Park, "Effect of cultural competence education for the nurse," Multicultural Education Studies, vol.6, no.2, pp.115-133, (2013)

[15] D. H. Chae and C. Y. Lee, "Development and psychometric evaluation of the Korean version of the cultural competence scale for clinical nurses," Asian Nursing Research, vol.8, pp.305-312, (2014)

[16] M. R. Jeffreys, "Teaching cultural competence in nursing and health care (2nd ed)," New York, NY: Springer Publishing Company, (2010)

[17] A. C. Brathwaite and B. Majumdar, "Evaluation of a cultural competence educational programme," Issues and Innovations in Nursing Education, vol.53, no.4, pp.470-479, (2006)

[18] M. K. Douglas, J. U. Pierce, M. Rosenkoetter, D. Pacquiao, L. C. Callister, and M. HattarPollara, "Standards of practice for culturally competent nursing care: 2011 update," Journal of Transcultural Nursing, vol.22, no.4, pp.317-333, (2011)

[19] American Association of Colleges of Nursing, "Tool kit resources for cultural competent education for baccalaureate nurses," Washington, DC: Author, (2008)

[20] [20] E. H. Peek and C. S. Park, "Effects of a multicultural education program on the cultural competence, empathy, and self-efficacy of nursing students," Journal of Korean Academy of Nursing, vol.43, no.5, pp.690-696, (2013)

[21] H. I. Cho Chung, S. Y. Han, and S. H. Seo, "Effects of a cultural competence educational program for nursing students," Journal of Korean Academy of Nursing Administration, vol.23, no.4, pp.684-693, (2017)

[22] S. Y. Han and H. I. Cho Chung, "Development of cultural competence scale for nursing students," Journal of Korean Academy of Nursing, vol.45, no.5, pp.684-693, (2015)

[23] S. Y. Han, "Factors influencing the cultural competence in nursing students," Journal of Learner-Centered Curriculum and Instruction, vol.16, no.4, pp.73-86, (2016)

[24] D. H. Chae, Y. H. Park, K. H. Kang, and T. H. Lee, "A study on affecting cultural competency of cultural competence of general hospital nurses," Journal of Korean Academy of Nursing Administration, vol.18, no.1, pp.76-86, (2012)

[25] S. Y. Yang, H. N. Lim, and J. H. Lee, "The study on the relationship between cultural competency and empathy of nursing students," Journal of Korean Academic Society of Nursing Education, vol.19, no.2, pp.183-193, (2013)

[26] J. S. Park, "Study on cultural competence of nurses working in a general hospital," M.S. thesis, Keimyung University, (2011)

[27] Y. R. Cha, "Factor related to cultural competence among baccalaureate nursing students in Korea," M.S. thesis, Ewha Womans University, (2014)

[28] M. S. Park and Y. R. Kweon, "Effects of a cultural competence promotion program for multicultural maternity nursing care: case-based small group learning," Journal of Korean Academy of Nursing, vol.43, no.5, pp.626-635, (2013)

[29] S. H. Kim, "Development of education program for cultural competence in nursing for nursing students and its effects," Journal of Korean Academic Society of Nursing Education, vol.19, no.4, pp.580-593, (2013)

[30] R. Benkert, T. Templin, S. M. Schim, A. Z. Doorenbos, and S. E. Bell, "Testing a multi-group model of culturally competent behaviors among underrepresented nurse practitioners," Research in Nursing \& Health, vol.34, no.4, pp.327-341, (2011)

[31] A. Cooper-Brathwaite, "Evaluation of a cultural competence course," Journal of Transcultural Nursing, vol.16, no.4, pp.361-369, (2005) 
[32] S. D. Hwang, H. Im and S. H. Yun, "Testing the effectiveness of multicultural education program," Korean Journal of Social Welfare, vol.64, no.1, pp.125-150, (2012)

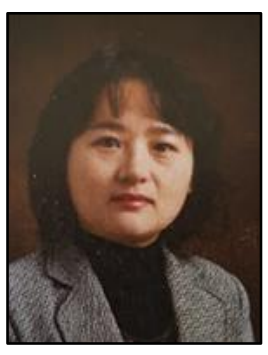

\section{Mi-Jung Choi}

Chosun Nursing College Associate professor B.A. Gwangju National Education University M.A. Gwangju National Education University Ph.D. Korea National University of Education Post. Doctoral course. Saint John's University Research field

Creativity \& Curriculum

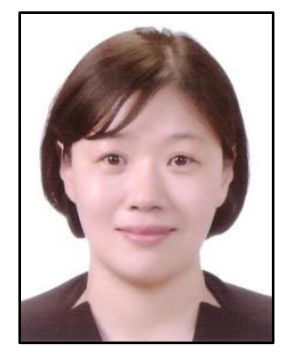

\section{Seokyoung, Han}

Chosun Nursing College Assistant professor

B.A. Christian College of Nursing

M.A. Chonnam National University

Ph.D. Chonnam National University

Research field

- Cultural Competence

- Pediatrics care programs 
Effects of the Global Multicultural Nursing Competency Enhancement Program on the Cultural Competence of Nursing College Students

This page is empty by intention. 